\title{
Discrete Spherical Harmonic Transforms: Numerical Preconditioning and Optimization
}

\author{
J.A. Rod Blais \\ Department of Geomatics Engineering \\ Pacific Institute for the Mathematical Sciences \\ University of Calgary, Calgary, AB, T2N 1N4, Canada \\ blais@ucalgary.ca, \\ www. ucalgary.ca/ blais
}

\begin{abstract}
Spherical Harmonic Transforms (SHTs) which are essentially Fourier transforms on the sphere are critical in global geopotential and related applications. Among the best known strategies for discrete SHTs are Chebychev quadratures and least squares. The numerical evaluation of the Legendre functions are especially challenging for very high degrees and orders which are required for advanced geocomputations. The computational aspects of SHTs and their inverses using both quadrature and least-squares estimation methods are discussed with special emphasis on numerical preconditioning that guarantees reliable results for degrees and orders up to 3800 in REAL $* 8$ or double precision arithmetic. These numerical results of spherical harmonic synthesis and analysis using simulated spectral coefficients are new and especially important for a number of geodetic, geophysical and related applications with ground resolutions approaching $5 \mathrm{~km}$.
\end{abstract}

\section{Introduction}

On the spherical Earth as on the celestial sphere, array computations can be done for regional and global domains using planar and spherical formulations. Spherical quadratures and least-squares estimation are used to convert continuous integral formulations into summations over data lattices. Spherical topologies are quite different from planar ones and these have important implications in the computational aspects of array data processing.

Spherical geocomputations for regional domains of even continental extents can be reduced to planar computations and under assumptions of stationarity or shift invariance, discrete array computations can be optimized using Fast Fourier Transforms (FFTs). Specifically, convolution operations for filtering and other data processing applications thereby require only $\mathrm{O}(\mathrm{N} \log \mathrm{N})$ instead of $\mathrm{O}\left(\mathrm{N}^{2}\right)$ operations for $\mathrm{N}$ data in one dimension, $\mathrm{O}\left(\mathrm{N}^{2} \log \mathrm{N}\right)$ instead of $\mathrm{O}\left(\mathrm{N}^{4}\right)$ operations for $\mathrm{NxN}$ data in two dimensions, and so on.

For global appplications, Gaussian, equiangular and other similar regular grids can be used for spherical quadratures and discrete convolutions. Various quadrature strategies are available in the literature going back to Gauss and Neumann, in addition to least-squares estimation techniques (e.g. $[8,16])$. Other approaches have also been 
used for discretization and analysis of functions on the sphere using triangular and curvilinear tesselations based on inscribed regular polytopes (see e.g. [2]). Depending on the applications, these strategies may be preferable to the equiangular ones which will be discussed in the following.

The associated Legendre functions for high degrees and orders are computationally very challenging. Without any normalization, one can hardly compute SHTs of degrees and orders over 50 or so in REAL*8 or double precision arithmetic. With proper normalization such as the geodetic one used in the following computations, one can achieve degrees and orders to around 1800 in REAL*8 or double precision arithmetic [4] and over 3600 in REAL*16 or quadruple precision arithmetic [5,6]. With latitudebased preconditioning, [13,14,19] achieved about 2700. With Clenshaw's approach, similar results can be achieved for appropriately decreasing spectral coefficients such as with the EGM06 model coefficients of degree and order 2190 [7,13].

The following shows that with proper numerical preconditioning independent of the latitude, the Legendre functions can be evaluated reliably for degrees and orders over 3800 in REAL*8 or double precision arithmetic. This is demonstrated explicitly in synthesis and analysis computations using unit spectral coefficients with equiangular grids that do not include the poles. In order words, the previously published results $[5,6]$ using Chebychev quadrature and least squares can be extended to degrees and orders over 3800 working in REAL*8 or double precision arithmetic. This is very important for numerous applications in geocomputations for ground resolutions of about $5 \mathrm{~km}$.

\section{Continuous and Discrete SHTs}

The orthogonal or Fourier expansion of a function $f(\theta, \lambda)$ on the sphere $\mathbf{S}^{2}$ is given by

$$
\mathrm{f}(\theta, \lambda)=\sum_{\mathrm{n}=0}^{\infty} \sum_{|\mathrm{m}| \leq \mathrm{n}} \mathrm{f}_{\mathrm{n}, \mathrm{m}} \mathrm{Y}_{\mathrm{n}}^{\mathrm{m}}(\theta, \lambda)
$$

using colatitude $\theta$ and longitude $\lambda$, where the basis functions $\mathrm{Y}_{\mathrm{n}}^{\mathrm{m}}(\theta, \lambda)$ are called the spherical harmonics of degree $n$ and order $m$. In particular, the Fourier or spherical harmonic coefficients appearing in the preceding expansion are obtained as inner products

$$
\mathrm{f}_{\mathrm{n}, \mathrm{m}}=\int_{\mathrm{s}^{2}} \mathrm{f}(\theta, \lambda) \overline{\mathrm{Y}}_{\mathrm{n}}^{\mathrm{m}}(\theta, \lambda) \mathrm{d} \sigma
$$

with the overbar denoting the complex conjugate with $d \sigma$ denoting the standard rotation invariant measure $\mathrm{d} \sigma=\sin \theta \mathrm{d} \theta \mathrm{d} \lambda$ on $\mathbf{S}^{2}$. In most practical applications, the functions $f(\theta, \lambda)$ are band-limited in the sense that only a finite number of those coefficients are nonzero, i.e. $\mathrm{f}_{\mathrm{n}, \mathrm{m}} \equiv 0$ for all degrees $\mathrm{n} \geq \mathrm{N}$ and orders $|\mathrm{m}| \leq \mathrm{n}$. Hence, using the regular equiangular grid $\theta_{\mathrm{j}}=\mathrm{j} \pi / \mathrm{J}$ and $\lambda_{\mathrm{k}}=\mathrm{k} 2 \pi / \mathrm{K}, \mathrm{j}=0, \ldots, \mathrm{J}-1, \mathrm{k}=0, \ldots, \mathrm{K}-1$, with $\mathrm{J}$ and $\mathrm{K}$ to be specified later on, spherical harmonic synthesis can be formulated as

$$
\mathrm{f}\left(\theta_{\mathrm{j}}, \lambda_{\mathrm{k}}\right)=\sum_{\mathrm{n}=0}^{\mathrm{N}-1} \sum_{|\mathrm{m}| \leq \mathrm{n}} \mathrm{f}_{\mathrm{n}, \mathrm{m}} \mathrm{Y}_{\mathrm{n}}^{\mathrm{m}}\left(\theta_{\mathrm{j}}, \lambda_{\mathrm{k}}\right)
$$


and using some appropriate spherical quadrature, the corresponding spherical harmonic analysis can be formulated as

$$
\mathrm{f}_{\mathrm{n}, \mathrm{m}}=\sum_{\mathrm{j}=0}^{\mathrm{J}-1} \sum_{\mathrm{k}=0}^{\mathrm{K}-1} \mathrm{q}_{\mathrm{j}} \mathrm{f}\left(\theta_{\mathrm{j}}, \lambda_{\mathrm{k}}\right) \overline{\mathrm{Y}}_{\mathrm{n}}^{\mathrm{m}}\left(\theta_{\mathrm{j}}, \lambda_{\mathrm{k}}\right)
$$

for quadrature weights $\mathrm{q}_{\mathrm{j}}$ as discussed by various authors e.g. [9,16,3].

The usual geodetic spherical harmonic formulation is given as

$$
\mathrm{f}(\theta, \lambda)=\sum_{\mathrm{n}=0}^{\infty} \sum_{\mathrm{m}=0}^{\mathrm{n}}\left[\tilde{\mathrm{c}}_{\mathrm{nm}} \cos \mathrm{m} \lambda+\tilde{\mathrm{s}}_{\mathrm{nm}} \sin \mathrm{m} \lambda\right] \tilde{\mathrm{P}}_{\mathrm{nm}}(\cos \theta)
$$

where

$$
\left\{\begin{array}{c}
\tilde{\mathrm{c}}_{\mathrm{nm}} \\
\tilde{\mathrm{s}}_{\mathrm{nm}}
\end{array}\right\}=\frac{1}{4 \pi} \int_{\mathrm{s}^{2}} \mathrm{f}(\theta, \lambda)\left\{\begin{array}{c}
\cos \mathrm{m} \lambda \\
\sin \mathrm{m} \lambda
\end{array}\right\} \tilde{\mathrm{P}}_{\mathrm{nm}}(\cos \theta) \mathrm{d} \sigma
$$

with the geodetically normalized Legendre functions $\tilde{\mathrm{P}}_{\mathrm{nm}}(\cos \theta)$ expressed in terms of the usual spherical harmonics $\mathrm{Y}_{\mathrm{n}}^{\mathrm{m}}(\theta, \lambda)$ (see e.g. [11] and [3] for details). The tilde “ " will be used to indicate geodetic normalization in the following.

Explicitly, using the geodetic formulation and convention, one has for synthesis,

$$
\mathrm{f}(\theta, \lambda)=\sum_{\mathrm{n}=0}^{\mathrm{N}-1} \sum_{\mathrm{m}=0}^{\mathrm{n}}\left[\tilde{\mathrm{c}}_{\mathrm{nm}} \cos \mathrm{m} \lambda+\tilde{\mathrm{s}}_{\mathrm{nm}} \sin \mathrm{m} \lambda\right] \tilde{\mathrm{P}}_{\mathrm{nm}}(\cos \theta)
$$

and for analysis, using complex analysis,

$$
\begin{aligned}
\tilde{\mathrm{c}}_{\mathrm{nm}}+\tilde{\mathrm{is}}_{\mathrm{nm}} & =\frac{1}{4 \pi} \int_{0}^{2 \pi} \int_{0}^{\pi} \mathrm{f}(\theta, \lambda)(\cos \mathrm{m} \lambda+\mathrm{i} \sin \mathrm{m} \lambda) \tilde{\mathrm{P}}_{\mathrm{nm}}(\cos \theta) \sin \theta \mathrm{d} \theta \mathrm{d} \lambda \\
& =\int_{0}^{\pi}\left[\mathrm{u}_{\mathrm{m}}(\theta)+\mathrm{iv}_{\mathrm{m}}(\theta)\right] \tilde{\mathrm{P}}_{\mathrm{nm}}(\cos \theta) \sin \theta \mathrm{d} \theta
\end{aligned}
$$

where

$$
u_{m}(\theta)+i v_{m}(\theta)=\frac{1}{4 \pi} \int_{0}^{2 \pi} f(\theta, \lambda)(\cos m \lambda+i \sin m \lambda) d \lambda
$$

which is simply the parallel-wise Fourier transform of the data.

Hence using data equispaced in longitude and the corresponding Discrete Fourier Transform (DFT) and Inverse DFT (IDFT), one can write for each parallel,

$$
\left\{\mathrm{f}\left(\theta, \lambda_{\mathrm{k}}\right)\right\} \stackrel{\mathrm{DFT}}{\longrightarrow}\left\{\mathrm{u}_{\mathrm{m}}(\theta)+\mathrm{iv}_{\mathrm{m}}(\theta)\right\} \stackrel{\mathrm{IDFT}}{\longrightarrow}\left\{\mathrm{f}^{\prime}\left(\theta, \lambda_{\mathrm{k}}\right)\right\}
$$

and correspondingly, for each meridian, with some appropriate Chebychev Quadrature (CQ) or Least Squares (LS) to be described explicitly below,

$$
\left\{\tilde{\mathrm{c}}_{\mathrm{nm}}+\mathrm{i} \tilde{\mathrm{s}}_{\mathrm{nm}}\right\} \stackrel{\text { SYNTHESIS }}{\longrightarrow}\left\{\mathrm{u}_{\mathrm{m}}(\theta)+\mathrm{iv}_{\mathrm{m}}(\theta)\right\} \stackrel{\mathrm{CQ} \text { or LS }}{\longrightarrow}\left\{\tilde{\mathrm{c}}_{\mathrm{nm}}^{\prime}+\mathrm{is}_{\mathrm{nm}}^{\prime}\right\}
$$

in which the Synthesis is only partial, i.e. in the Fourier domain. Notice that in general for band limit $\mathrm{N}, \mathrm{N}$ rows of equilatitude data are required with $\mathrm{LS}$ while $2 \mathrm{~N}$ rows of equispaced equilatitude data are required with the $\mathrm{CQ}$, and at least $2 \mathrm{~N}$ equispaced data are required for DFT per parallel (see e.g. [5,6] for more discussion). 
Furthermore, for data grids with $\Delta \theta=\Delta \lambda$ which are often required in practice, such as $\left\{\left(\theta_{\mathrm{j}}, \lambda_{\mathrm{k}}\right) \mid \theta_{\mathrm{j}}=\mathrm{j} \pi / \mathrm{N}, \lambda_{\mathrm{k}}=\mathrm{k} \pi / \mathrm{N} ; \mathrm{j}=0,1, \ldots, \mathrm{N}-1, \mathrm{k}=0,1, \ldots, 2 \mathrm{~N}-1\right\}$

for LS, or

$$
\left\{\left(\theta_{\mathrm{j}}, \lambda_{\mathrm{k}}\right) \mid \theta_{\mathrm{j}}=\mathrm{j} \pi / 2 \mathrm{~N}, \lambda_{\mathrm{k}}=\mathrm{k} \pi / 2 \mathrm{~N} ; \mathrm{j}=0,1, \ldots, 2 \mathrm{~N}-1, \mathrm{k}=0,1, \ldots, 4 \mathrm{~N}-1\right\}
$$

for $\mathrm{CQ}$, the poles can be excluded with a shift in latitude

$$
\left\{\left(\theta_{\mathrm{j}}, \lambda_{\mathrm{k}}\right) \mid \theta_{\mathrm{j}}=(\mathrm{j}+1 / 2) \pi / \mathrm{N}, \lambda_{\mathrm{k}}=\mathrm{k} \pi / \mathrm{N} ; \mathrm{j}=0,1, \ldots, \mathrm{N}-1, \mathrm{k}=0,1, \ldots, 2 \mathrm{~N}-1\right\}
$$

and correspondingly,

$$
\left\{\left(\theta_{\mathrm{j}}, \lambda_{\mathrm{k}}\right) \mid \theta_{\mathrm{j}}=(\mathrm{j}+1 / 2) \pi / 2 \mathrm{~N}, \lambda_{\mathrm{k}}=\mathrm{k} \pi / 2 \mathrm{~N} ; \mathrm{j}=0,1, \ldots, 2 \mathrm{~N}-1, \mathrm{k}=0,1, \ldots, 4 \mathrm{~N}-1\right\}
$$

which allow the use of hemispherical symmetries in the associated Legendre functions

$$
\mathrm{P}_{\mathrm{nm}}(\cos (\pi-\theta))=(-1)^{\mathrm{n}+\mathrm{m}} \mathrm{P}_{\mathrm{nm}}(\cos \theta)
$$

Notice that these data grids with $\Delta \theta=\Delta \lambda$ have $\mathrm{Nx} 2 \mathrm{~N}$ and $2 \mathrm{Nx} 4 \mathrm{~N}$ quantities and $\mathrm{N}^{2}$ spectral coefficients for band limit N. More details with simulated results can be found in $[5,6]$.

\section{Numerical Preconditioning and Optimization}

The numerical evaluation of the associated Legendre functions $P_{n m}(\cos \theta)$ for colatitude $\theta$, is very challenging for high degrees $n$ and orders $m$. To see this, one only has to evaluate the diagonal terms $\mathrm{P}_{\mathrm{nn}}\left(\cos 1^{\circ}\right)$ for $\mathrm{n}=1,2, \ldots, 100, \ldots$, using different normalizations. Furthermore, there are several strategies with recursions in $\mathrm{n}$ and $\mathrm{m}$ and these are far from being numerically equivalent (see e.g. $[1,3,14]$ ).

The geodetically normalized associated Legendre functions $\tilde{\mathrm{P}}_{\mathrm{nm}}(\cos \theta)$ are computed as a lower triangular matrix with the rows corresponding to the degrees $\mathrm{n}$ and the columns corresponding to the orders $\mathrm{m}$. With the initialization for degrees and orders 0 and $1, \quad \tilde{\mathrm{P}}_{00}(\cos \theta)=1, \tilde{\mathrm{P}}_{10}(\cos \theta)=\sqrt{3} \cos \theta$ and $\tilde{\mathrm{P}}_{11}(\cos \theta)=\sqrt{3} \sin \theta$, the diagonal terms $\tilde{\mathrm{P}}_{\mathrm{nn}}(\cos \theta)=\sqrt{(2 \mathrm{n}+1) / 2 \mathrm{n}} \sin \theta \tilde{\mathrm{P}}_{\mathrm{n}-1, \mathrm{n}-1}(\cos \theta)$ and the subdiagonal terms $\tilde{\mathrm{P}}_{\mathrm{n}, \mathrm{n}-1}(\cos \theta)=\sqrt{2 \mathrm{n}+1} \cos \theta \tilde{\mathrm{P}}_{\mathrm{n}-1, \mathrm{n}-1}(\cos \theta)$ computed recursively. The remaining terms for $n \geq 2$ and $n-2 \geq m \geq 0$ are evaluated with a three-term formula

$$
\cos \theta \tilde{\mathrm{P}}_{\mathrm{nm}}(\cos \theta)=\alpha_{\mathrm{nm}} \tilde{\mathrm{P}}_{\mathrm{n}-1, \mathrm{~m}}(\cos \theta)+\alpha_{\mathrm{n}+1, \mathrm{~m}} \tilde{\mathrm{P}}_{\mathrm{n}+1, \mathrm{~m}}(\cos \theta)
$$

with $\alpha_{n m}=\sqrt{(n-m)(n+m) /(2 n-1)(2 n+1)}$ as detailed in [18], based on [10, 15]. With this approach, degrees and orders up to 1800 or so have been achieved quite reliably with $\mathrm{REAL} * 8$ or double precision and over 3600 with $\mathrm{REAL} * 16$ or quadruple precision arithmetic [5,6].

Using IEEE standards for floating point arithmetic on personal and similar computers, the EXPONENT limits for real, double and quadruple precisions are as follows:

\begin{tabular}{|c|c|c|}
\hline Variable Type & $\begin{array}{c}\text { Minimum EXPO- } \\
\text { NENT }\end{array}$ & Maximum EXPONENT \\
\hline REAL $* 4$ & -125 & 128 \\
\hline REAL $* 8$ & -1021 & 1024 \\
\hline REAL 16 & -16381 & 16384 \\
\hline
\end{tabular}


Hence to avoid underflow problems in computing $\tilde{\mathrm{P}}_{\mathrm{nm}}(\cos \theta)$, a simple strategy is to bias the EXPONENT of the corresponding variable by a large number and remove the bias once the computations are done. Specifically, using personal and similar computers with REAL*8 or double precision arithmetic, a bias of 1000 was applied to the EXPONENT of the variable in question and the resulting numerical computations are very stable. More details are included in the next Section.

This strategy of biasing the EXPONENT is independent of the colatitude $\theta$ which is very important in this context. The literature on computing very high degree spherical harmonics contains numerous strategies such as using the Clenshaw summation approach [13], preconditioning the variable with $10^{280} \sin \theta$ in SHTools [19], modifying the recursion to avoid high powers of $\sin \theta$ [14], and others. These modifications have been reported to achieve degrees around 2700-2800 in synthesis computations [13, 14] and in synthesis and analysis [19]. Notice that in synthesis only computations, it is only necessary to avoid underflows and set the corresponding incremental contributions to zero while in synthesis and analysis computations, the numerical recovery of the input spectral coefficients is expected in addition to avoiding possible underflows.

\section{Numerical Experimentation}

To test the numerical preconditioning procedure, consider the following

$$
\left\{\tilde{\mathrm{c}}_{\mathrm{nm}}+\mathrm{is}_{\mathrm{nm}}\right\} \stackrel{\text { SYNTHESIS }}{\longrightarrow}\left\{\mathrm{u}_{\mathrm{m}}(\theta)+\mathrm{iv}_{\mathrm{m}}(\theta)\right\} \stackrel{\mathrm{CQ} \text { or LS }}{\longrightarrow}\left\{\tilde{\mathrm{c}}_{\mathrm{nm}}^{\prime}+\mathrm{i}_{\mathrm{nm}}^{\prime}\right\}
$$

with the partial SYNTHESIS in the Fourier domain as

$$
\mathrm{u}_{\mathrm{m}}(\theta)+i \mathrm{v}_{\mathrm{m}}(\theta)=\sum_{\mathrm{n}=\mathrm{m}}^{\mathrm{N}-1}\left(\tilde{\mathrm{c}}_{\mathrm{nm}}+\mathrm{i} \tilde{\mathrm{s}}_{\mathrm{nm}}\right) \tilde{\mathrm{P}}_{\mathrm{nm}}(\cos \theta)
$$

for $2 \mathrm{~N}$ isolatitudes with $\Delta \theta=\pi / 2 \mathrm{~N}$ for CQ and $\mathrm{N}$ isolatitudes with $\Delta \theta=\pi / \mathrm{N}$ for LS. In longitude, $2 \mathrm{~N}$ equispaced points with $\Delta \lambda=\pi / 2 \mathrm{~N}$ for both CQ and LS in the following experimentation. A shift in latitude of the grids by half $\Delta \theta$ has been implemented to exclude the poles.

The Chebychev quadrature is as follows

$$
\tilde{\mathrm{c}}_{\mathrm{nm}}^{\prime \prime}+\mathrm{i} \tilde{\mathrm{s}}_{\mathrm{nm}}^{\prime \prime}=\sum_{\mathrm{j}=0}^{2 \mathrm{~N}-1} \mathrm{q}_{\mathrm{j}}\left(\mathrm{u}_{\mathrm{jm}}+\mathrm{i} \mathrm{v}_{\mathrm{jm}}\right) \tilde{\mathrm{P}}_{\mathrm{nm}}\left(\cos \theta_{\mathrm{j}}\right)
$$

with $\mathrm{u}_{\mathrm{jm}} \equiv \mathrm{u}_{\mathrm{m}}\left(\theta_{\mathrm{j}}\right)$ and $\mathrm{v}_{\mathrm{jm}} \equiv \mathrm{v}_{\mathrm{m}}\left(\theta_{\mathrm{j}}\right)$, and CQ weights

$$
\mathrm{q}_{\mathrm{j}}=\frac{1}{\mathrm{~N}} \sin ((\mathrm{j}+1 / 2) \pi / 2 \mathrm{~N}) \sum_{\mathrm{h}=0}^{\mathrm{N}-1} \frac{1}{2 \mathrm{~h}+1} \sin ((2 \mathrm{~h}+1)(\mathrm{j}+1 / 2) \pi / 2 \mathrm{~N})
$$

with $\mathrm{q}_{2 \mathrm{~N}-\mathrm{j}}=\mathrm{q}_{\mathrm{j}}$ for $\mathrm{j}=0,1, \ldots, \mathrm{N}-1$ by hemispherical symmetry. These computations are roughly $\mathrm{O}\left(\mathrm{N}^{3}\right)$ for degree $\mathrm{N}$.

The least-squares formulation per degree $\mathrm{m}$ is as follows 


$$
\sum_{n=m}^{N-1} \tilde{P}_{n m}\left(\cos \theta_{j}\right)\left(\tilde{\mathrm{c}}_{\mathrm{nm}}^{\prime \prime \prime}+\mathrm{i}_{\mathrm{nm}}^{\prime \prime \prime}\right)=\mathrm{u}_{\mathrm{m}}\left(\theta_{\mathrm{j}}\right)+\mathrm{iv}_{\mathrm{m}}\left(\theta_{\mathrm{j}}\right)
$$

with isolatitudes

$$
\theta_{\mathrm{j}}=(\mathrm{j}+1 / 2) \pi / \mathrm{N}=(2 \mathrm{j}+1) \pi / 2 \mathrm{~N} \quad \text { for } \mathrm{j}=0,1, \ldots, \mathrm{N}-1 .
$$

The least-squares computations for $\tilde{\mathrm{c}}_{\mathrm{nm}}^{\prime \prime \prime}+\mathrm{is}_{\mathrm{nm}}^{\prime \prime \prime}$ per degree $\mathrm{m}$ are obviously very demanding and roughly $\mathrm{O}\left(\mathrm{N}^{4}\right)$. The elements in the corresponding normal matrices can be evaluated using the Christoffel-Darboux formula as shown in [18, Appendix B] based on [12], but this has not yet been implemented in the code yet.

Starting with simulated unit spectral coefficients, the reconstructed coefficients are compared with the input coefficients and Root-Mean-Square (RMS) values are computed for various degrees and orders using both CQ and LS. The key results, all obtained in REAL*8 or double precision arithmetic, are shown in Table 1 for degrees and orders to 3800 . The results for degrees and orders to 1800 or so agree exactly with those REAL*8 or double precision results published in [5 and 6]. The second column of Table 1 are the RMS results on a Notebook (NB) while the third column are the RMS results on a desktop PC, both with AMD Athlon64 Dual-Core Processors. The RMS decreases to E-04 for 3900 with CQ but the situation is better using LS with E-13 for 3900 and E-09 for 4000. The numerical stability exhibited in Table 1 for synthesis/ analysis for degrees over 3000 has not previously been seen anywhere else in the literature.

Table 1. Numerical RMS Results for SYNTHESIS/ANALYSIS with simulated unit spectral coefficients on AMD64 NB and PC in REAL*8 or Double Precision arithmetic

\begin{tabular}{|c|c|c|}
\hline $\begin{array}{c}\text { Degrees } \\
\mathrm{N}\end{array}$ & $\begin{array}{c}\text { CQ RMS on AMD64 NB } \\
\text { (data grid: } 2 \mathrm{Nx} 2 \mathrm{~N} \text { ) }\end{array}$ & $\begin{array}{c}\text { LS RMS on AMD64 PC } \\
\text { (data grid: Nx2N) }\end{array}$ \\
\hline 1000 & $1.24557 \mathrm{E}-12$ & $5.53768 \mathrm{E}-14$ \\
\hline 2000 & $3.16427 \mathrm{E}-12$ & $1.13533 \mathrm{E}-13$ \\
\hline 3000 & $6.72616 \mathrm{E}-12$ & $1.67988 \mathrm{E}-13$ \\
\hline 3200 & $2.59890 \mathrm{E}-12$ & $1.66504 \mathrm{E}-13$ \\
\hline 3400 & $3.86647 \mathrm{E}-12$ & $1.65382 \mathrm{E}-13$ \\
\hline 3600 & $3.54980 \mathrm{E}-12$ & $1.64626 \mathrm{E}-13$ \\
\hline 3800 & $5.63723 \mathrm{E}-11$ & $2.08633 \mathrm{E}-13$ \\
\hline
\end{tabular}

\section{Concluding Remarks}

Considerable work has been done on solving the computational complexities, and enhancing the speed of calculation of spherical harmonic transforms for different equiangular grids. The numerical problems of evaluating the associated Legendre functions for very high degrees and orders have been solved using numerical preconditioning in terms of the EXPONENT of the corresponding variable. Explicitly, using 
simulated unit spectral coefficients for degrees and orders up to 3800, partial synthesis and analysis lead to RMS errors of orders $10^{-12}-10^{-13}$. When starting with simulated spherical harmonic coefficients corresponding to $1 /$ degree $^{2}$, the previously results can be expected to improve by a couple of orders of magnitude, as experienced in previous experimental work $[4,5,6]$. The latter simulations would perhaps be more indicative of the expected numerical accuracies in practice.

As enormous quantities of data are involved the intended gravity field applications, parallel and grid computations are imperative for these applications. Preliminary experimentation with parallel processing has already been done [17] and these REAL*8 or double precision results can readily be duplicated in parallel environments.

Acknowledgement. The author would like to acknowledge the sponsorship of the Natural Science and Engineering Research Council in the form of a Research Grant on Computational Tools for the Geosciences. Special thanks are also expressed to Dr. M. Soofi, Post Doctoral Fellow (2005-2006) in Geomatics Engineering and Geoscience, University of Calgary, for helping with the optimization of the code for different computer platforms.

\section{References}

1. Adams, J.C., Swarztrauber, P.N.: SPHEREPACK 2.0: A Model Development Facility (1997), http: / /www.scd.ucar.edu/softlib/SPHERE.html

2. Blais, J.A.R.: Optimal Spherical Triangulation for Global Multiresolution Analysis and Synthesis. In: The 2007, Fall Meeting of the American Geophysical Union in San Francisco, CA (2007)

3. Blais, J.A.R., Provins, D.A.: Spherical Harmonic Analysis and Synthesis for Global Multiresolution Applications. Journal of Geodesy 76(1), 29-35 (2002)

4. Blais, J.A.R., Provins, D.A.: Optimization of Computations in Global Geopotential Field Applications. In: Sloot, P.M.A., Abramson, D., Bogdanov, A.V., Gorbachev, Y.E., Dongarra, J., Zomaya, A.Y. (eds.) ICCS 2003. LNCS, vol. 2658, pp. 610-618. Springer, Heidelberg (2003)

5. Blais, J.A.R., Provins, D.A., Soofi, M.A.: Spherical Harmonic Transforms for Discrete Multiresolution Applications. Journal of Supercomputing 38, 173-187 (2006)

6. Blais, J.A.R., Provins, D.A., Soofi, M.A.: Optimization of Spherical Harmonic Transform Computations. In: Sunderam, V.S., van Albada, G.D., Sloot, P.M.A., Dongarra, J. (eds.) ICCS 2005. LNCS, vol. 3514, pp. 74-81. Springer, Heidelberg (2005)

7. Blais, J.A.R., Soofi, M.A.: Optimization of Discrete Spherical Harmonic Transforms and Applications. Poster Presentation at the 2006 Fall Meeting of the American Geophysical Union in San Francisco, CA (2006)

8. Colombo, O.: Numerical Methods for Harmonic Analysis on the Sphere. Report no. 310, Department of Geodetic Science and Surveying, The Ohio State University (1981)

9. Driscoll, J.R., Healy Jr., D.M.: Computing Fourier Transforms and Convolutions on the 2Sphere. Advances in Applied Mathematics 15, 202-250 (1994)

10. Gradshteyn, I.S., Ryzhik, I.M.: Tables of Integrals, Series and Products. Academic Press, London (1980)

11. Heiskanen, W.A., Moritz, H.: Physical Geodesy, p. 363. W.H. Freeman, San Francisco (1967) 
12. Hildebrand, F.B.: Introduction to Numerical Analysis. McGraw-Hill, New York (1956)

13. Holmes, S.A., Featherstone, W.E.: A Unified Approach to the Clenshaw Summation and the Recursive Computation of Very High Degree and Order Normalised Associated Legendre Functions. Journal of Geodesy 76, 279-299 (2002)

14. Jekeli, C., Lee, J.K., Kwon, J.H.: On the Computation and Approximation of Ultra-HighDegree Spherical Harmonic Series. Journal of Geodesy 81(9), 603-615 (2007)

15. Paul, M.K.: Recurrence Relations for Integrals of Associated Legendre Functions. Bulletin Geodesique 52, 177-190 (1978)

16. Sneeuw, N.: Global Spherical Harmonic Analysis by Least-Squares and Numerical Quadrature Methods in Historical Perspective. Geophys. J. Int. 118, 707-716 (1994)

17. Soofi, M.A., Blais, J.A.R.: Parallel Computations of Spherical Harmonic Transforms, Oral Presentation at the 2005 Annual Meeting of the Canadian Geophysical Union, Banff, Alberta, Canada (2005)

18. Swarztrauber, P.N., Spotz, W.F.: Generalized Discrete Spherical Harmonic Transforms. J. Comp. Phys. 159, 213-230 (2000)

19. Wieczorek, M.: SHTOOLS: Tools for Working with Spherical Harmonics. Centre National de la Recherche Scientifique, Institut de Physique du Globe de Paris (2007), http: / www . ipgp. jussieu. fr/ wieczor/SHTOOLS/www/accuracy.html 